\title{
Diagnosis and model based identification of a coupling misalignment
}

\author{
P. Pennacchi* and A. Vania \\ Department of Mechanics, Politecnico di Milano, Via La Masa, 34, I-20156, Milano, Italy
}

Received 2 April 2004

Revised 26 October 2004

\begin{abstract}
This paper is focused on the application of two different diagnostic techniques aimed to identify the most important faults in rotating machinery as well as on the simulation and prediction of the frequency response of rotating machines. The application of the two diagnostics techniques, the orbit shape analysis and the model based identification in the frequency domain, is described by means of an experimental case study that concerns a gas turbine-generator unit of a small power plant whose rotor-train was affected by an angular misalignment in a flexible coupling, caused by a wrong machine assembling. The fault type is identified by means of the orbit shape analysis, then the equivalent bending moments, which enable the shaft experimental vibrations to be simulated, have been identified using a model based identification method. These excitations have been used to predict the machine vibrations in a large rotating speed range inside which no monitoring data were available. To the best of the authors' knowledge, this is the first case of identification of coupling misalignment and prediction of the consequent machine behaviour in an actual size rotating machinery. The successful results obtained emphasise the usefulness of integrating common condition monitoring techniques with diagnostic strategies.
\end{abstract}

Keywords: Rotor dynamics, coupling misalignment, diagnostics, fault identification

\section{Introduction}

The health analysis of rotating machines can be assured by permanent condition monitoring systems. However, to satisfy the main requirements of predictive maintenance, protective and condition monitoring techniques should be integrated with diagnostic methods aimed to identify the machine faults. Orbit shape analysis $[16,17]$ is one of the possible methods, which has proved to be effective in the field practice [6,23].

Furthermore, if the diagnostic method is model based, not only the type, but also the severity and the location of the fault can be identified [2-5,13,14,19,20,24,31]. Usually, the machine rotor train is modelled with Finite Element (FE) beams [15], while the dynamic stiffness of rolling bearings and of oil-film journal bearings is modelled with rotating speed dependent coefficients. Moreover, the foundation structure can be modelled by means of its modal parameters $[5,29]$, mechanical impedance matrices $[7,27]$ or lumped mass-damping-stiffness systems generally called pedestals $[5,8,9]$.

Usually, model-based fault identification methods allow the most important failures to be simulated with a suitable set of equivalent excitations [11,12]. In the case of the rotors, excitations are applied to the nodes of the finite elements by means of which the machine rotor-train is modelled [2,4,5,19,20,24]. Further, model-based techniques enable the machine frequency response caused by the identified faults to be evaluated. Therefore, it is possible to predict the machine dynamic behaviour also in operating conditions different from those for which experimental vibration data are available. This capability will be shown in this paper since the behaviour of the machine in

*Corresponding author: P. Pennacchi, Department of Mechanics, Politecnico di Milano, Via La Masa, 34, I-20156, Milano, Italy. Tel.: +39 0223998440; Fax: +39 0223998492; E-mail: paolo.pennacchi@polimi.it. 
presence of the fault is forecasted up to the operating speed during a run-up in presence of the fault. Sometimes, this strategy allows protective operating conditions to be determined so that rotating machines that are affected by a fault in an early stage of development could be operated for a longer time in safe conditions without requiring earlier maintenance actions formerly scheduled later on. Therefore, model-based methods can be a very useful tool to improve the effectiveness of the most common techniques used to assure the machine health surveillance.

The fault taken into account in this paper is one of the most common in rotating machinery that present coupling between the shafts, so that handbooks are also available to perform good alignments [32]. However, literature studies are mainly devoted to theoretical analysis $[25,28,30]$ using numerical simulation and to the validation of the theoretical assumptions on small scale test-rigs $[10,18,26]$. Some experimental results of coupling misalignment identification on a test-rig using wavelets are presented in [21,22].

This paper shows the results of the analysis of the experimental vibrations of a real machine whose rotor-train was affected by an angular misalignment in the flexible coupling between two shafts. This fault was caused by a wrong reassembling of the rotor-train accomplished after the conclusion of some important maintenance actions. A diagnostic method based on a model of the fully assembled machine is used to identify the amplitude of the bending moments by means of which the shaft vibrations induced by the coupling misalignment can be simulated.

The evidences of the fault were detected using an orbit shape analysis at very low rotating speeds during the initial stage of the first runup carried out after a machine outage. The fault symptoms highlighted by the shaft vibrations, as well as the results provided by the fault identification method, suggested to inspect only a limited part of the rotor-train. Owing to this a severe coupling misalignment was timely detected and the causes of this fault were removed. Moreover, the identified bending moments have been used to predict the shaft vibrations that would have occurred if the fault had not been timely detected during the first aborted runup.

Therefore, this paper shows how different diagnostics techniques can be combined each other to integrate successfully the results provided by permanent condition monitoring systems.

\section{Orbit shape analysis}

The first diagnostic method used in this paper is based on the analysis of the journal filtered orbits determined with a couple of radial and orthogonal (XY) vibration probes (Fig. 1). The analysis of the filtered orbits is obtained by evaluating the degree of ellipticity and the inclination angle of the major axis of the orbit with respect to the horizontal axis. The degree of ellipticity of the orbit is provided by the Shape and Directivity Index (SDI) which is defined as [16,17]:

$$
-1 \leqslant S D I=\begin{aligned}
& \left|r^{f}\right|-\left|r^{b}\right| \\
& \left|r^{f}\right|+\left|r^{b}\right|
\end{aligned} \leqslant 1
$$

where $r^{f}$ and $r^{b}$ are, respectively, the forward and backward harmonic components of the complex harmonic signal $p(t)$ of frequency $\omega$. The real and imaginary parts of the signal $p(t)$ are respectively the real vibration signals $x(t)$ and $y(t)$, filtered at frequency $\omega$, measured along the shaft in the two orthogonal directions $\xi$ and $\eta$ (see Fig. 1):

$$
x(t)=X e^{i\left(\omega t+\varphi_{x}\right)}, \quad y(t)=Y e^{i\left(\omega t+\varphi_{y}\right)}
$$

Therefore, the complex signal $p(t)$ can be written in polar form, using the Euler's formula, as:

$$
p(t)=x(t)+i y(t)=p^{f}(t)+i p^{b}(t)=r^{f} e^{i \omega t}+r^{b} e^{-i \omega t}
$$

The direct $x_{d}$ and the quadrature $x_{q}$ parts of the vibration signal $x(t)$ are defined by considering that the vibration signal $x(t)$ can be written as:

$$
x(t)=x_{d} \cos \omega t+x_{q} \sin \omega t
$$

and also as:

$$
\begin{aligned}
x(t) & =X \cos \left(\omega t+\varphi_{x}\right)=X \cos \omega t \cos \varphi_{x}-X \sin \omega t \sin \varphi_{x}=\left(X \cos \varphi_{x}\right) \cos \omega t+\left(-X \sin \varphi_{x}\right) \sin \omega t \\
& =x_{d} \cos \omega t+x_{q} \sin \omega t
\end{aligned}
$$




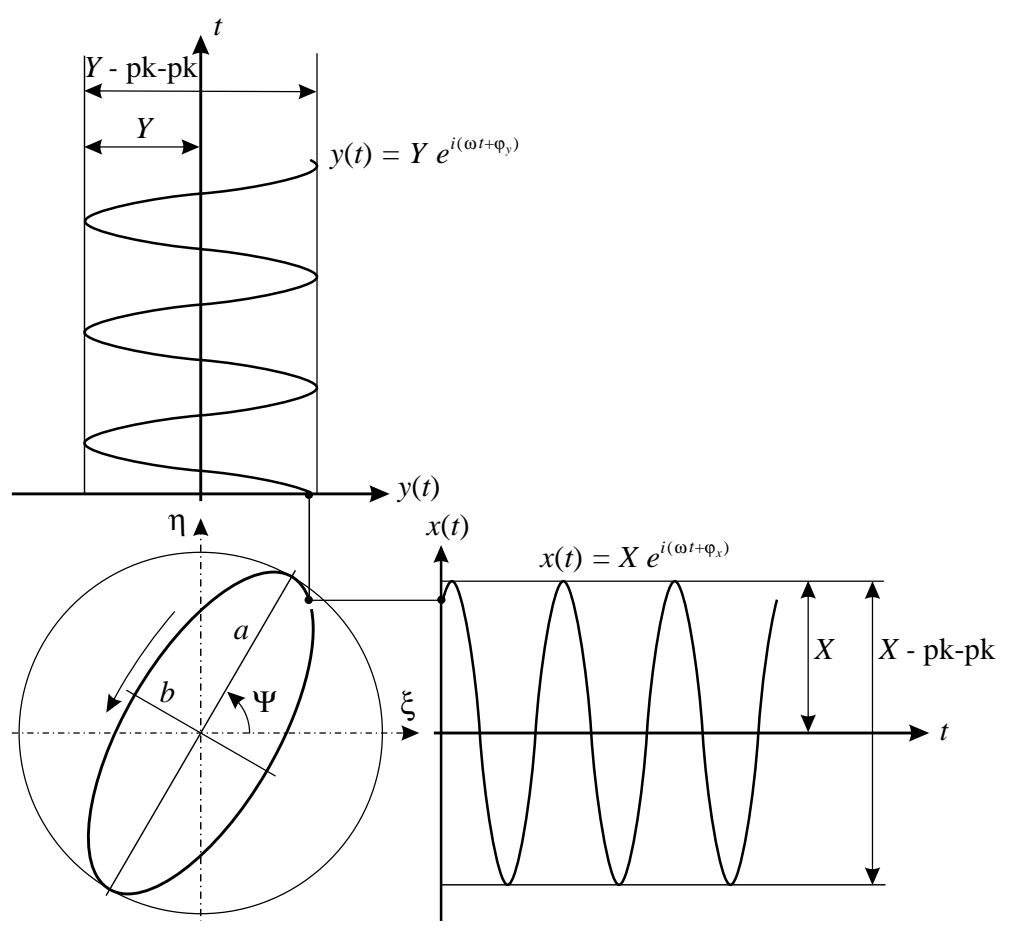

Fig. 1. Filtered orbit and vibration signals.

where:

$$
x_{d}=X \cos \varphi_{x}, \quad x_{q}=-X \sin \varphi_{x}
$$

Similarly, for the vibration signal $y(t)$ :

$$
y(t)=y_{d} \cos \omega t+y_{q} \sin \omega t
$$

and:

$$
\begin{aligned}
y(t) & =Y \cos \left(\omega t+\varphi_{y}\right)=Y \cos \omega t \cos \varphi_{y}-Y \sin \omega t \sin \varphi_{y} \\
& =\left(Y \cos \varphi_{y}\right) \cos \omega t+\left(-Y \sin \varphi_{y}\right) \sin \omega t=y_{d} \cos \omega t+y_{q} \sin \omega t
\end{aligned}
$$

So that direct $y_{d}$ and quadrature $y_{q}$ parts are given by:

$$
y_{d}=Y \cos \varphi_{y}, \quad y_{q}=-Y \sin \varphi_{y}
$$

Finally, the complex signal $p(t)$ can be rewritten in the following form:

$$
p(t)=\frac{1}{2}\left(\left(x_{d}+y_{q}\right)+i\left(y_{d}-x_{q}\right)\right) e^{i \omega t}+\frac{1}{2}\left(\left(x_{d}-y_{q}\right)+i\left(y_{d}+x_{q}\right)\right) e^{-i \omega t}
$$

Therefore:

$$
r^{f}=\frac{1}{2}\left(\left(x_{d}+y_{q}\right)+i\left(y_{d}-x_{q}\right)\right), \quad r^{b}=\frac{1}{2}\left(\left(x_{d}-y_{q}\right)+i\left(y_{d}+x_{q}\right)\right)
$$

Actually, by replacing Eqs (6) and (9) in (10), Eq. (3) is obtained again:

$$
\begin{aligned}
p(t) & ={ }_{2}^{1}\left\{\left(x_{d}+y_{q}\right)+i\left(y_{d}-x_{q}\right)\right\} e^{i \omega t}+{ }_{2}^{1}\left\{\left(x_{d}-y_{q}\right)+i\left(y_{d}+x_{q}\right)\right\} e^{-i \omega t} \\
& =\frac{1}{2}\{X \cos \varphi_{x} \underbrace{-}_{+i \cdot i} Y \sin \varphi_{y}+i Y \cos \varphi_{y}+i X \sin \varphi_{x}\} e^{i \omega t}
\end{aligned}
$$




$$
\begin{aligned}
& +{ }_{2}^{1}\{X \cos \varphi_{x} \underbrace{+}_{-i \cdot i} Y \sin \varphi_{y}+i Y \cos \varphi_{y}-i X \sin \varphi_{x}\} e^{-i \omega t}
\end{aligned}
$$

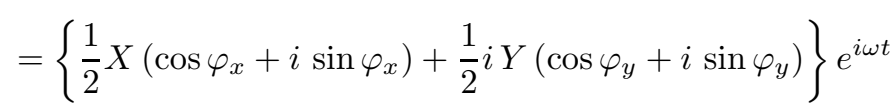

$$
\begin{aligned}
& +\left\{{ }_{2}^{1} X\left(\cos \varphi_{x}-i \sin \varphi_{x}\right)+{ }_{2}^{1} i Y\left(\cos \varphi_{y}-i \sin \varphi_{y}\right)\right\} e^{-i \omega t}
\end{aligned}
$$

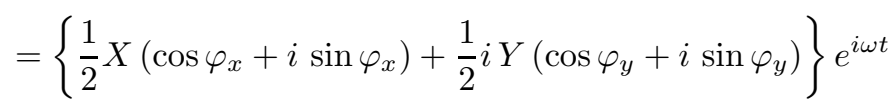

$$
\begin{aligned}
& +\left\{{ }_{2}^{1} X\left(\cos \left(-\varphi_{x}\right)+i \sin \left(-\varphi_{x}\right)\right)+{ }_{2}^{1} i Y\left(\cos \left(-\varphi_{y}\right)-i \sin \left(-\varphi_{y}\right)\right)\right\} e^{-i \omega t} \\
& ={ }_{2}^{1}\left\{X e^{i \varphi_{x}}+i Y e^{i \varphi_{y}}\right\} e^{i \omega t}+{ }_{2}^{1}\left\{X e^{-i \varphi_{x}}+i Y e^{-i \varphi_{y}}\right\} e^{-i \omega t} \\
& ={ }_{2}^{1} X e^{i\left(\omega t+\varphi_{x}\right)}+{ }_{2}^{1} i Y e^{i\left(\omega t+\varphi_{y}\right)}+{ }_{2}^{1} X e^{-i\left(\omega t+\varphi_{x}\right)}+{ }_{2}^{1} i Y e^{-i\left(\omega t+\varphi_{x}\right)} \\
& ={ }_{2}^{1} X e^{i \vartheta_{x}}+{ }_{2}^{1} X e^{-\vartheta_{x}}+{ }_{2}^{1} i Y e^{i \vartheta_{y}}+{ }_{2}^{1} i Y e^{-i \vartheta_{y}}=X \cos \vartheta_{x}+i Y \cos \vartheta_{y} \\
& ={ }_{2}^{1} X e^{i\left(\omega t+\varphi_{x}\right)}+{ }_{2}^{1} i Y e^{i\left(\omega t+\varphi_{y}\right)}+{ }_{2}^{1} X e^{-i\left(\omega t+\varphi_{x}\right)}+{ }_{2}^{1} i Y e^{-i\left(\omega t+\varphi_{y}\right)}
\end{aligned}
$$

The sign of the SDI index determines the directivity of the orbit while the absolute value of the SDI gives the degree of ellipticity. In fact, a positive unity value of the SDI indicates a forward circular motion while a negative unity value indicates a backward circular motion. A backward elliptic motion is associated with SDI values ranging from -1 to 0 while a forward elliptic motion is associated with SDI values ranging from 0 to 1 . When the SDI is null the orbit becomes a straight line. The inclination angle $\psi$ of the major axis of the ellipse with respect to the $x$ axis is given by:

$$
\psi={ }_{2}^{1} \begin{array}{cc}
\tan ^{-1} & 2\left(x_{d} y_{d}+x_{q} y_{q}\right) \\
y_{d}^{2}+y_{q}^{2}-\left(x_{d}^{2}+x_{q}^{2}\right)
\end{array}
$$

In addition to this, the amplitude of the major $a$ and minor $b$ axes of the elliptical orbit can be evaluated using:

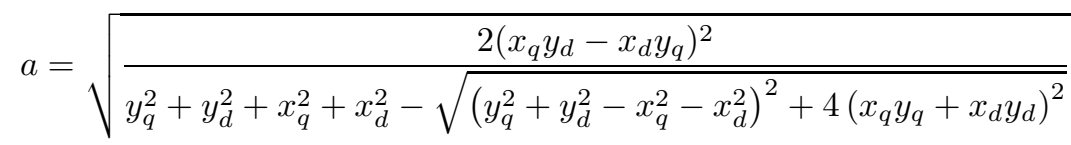

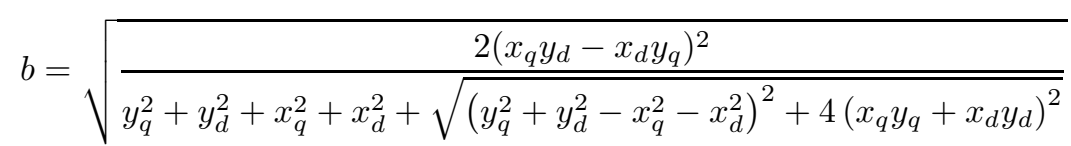

The shape and directivity index can be defined also as the ratio between the amplitudes of the minor axis $b$ and the major axis $a$ of the filtered orbit:

$$
\mathrm{SDI}=\begin{aligned}
& b \\
& a
\end{aligned}
$$

\section{Model based identification method in the frequency domain}

Some model-based methods aimed to identify faults in rotating machines are developed in the time domain [19, 24], however, the approach described in this paper, as well as most of the fault identification techniques, has been developed in the frequency domain [2-6]. 
The model-based technique used in this investigation to identify machine faults is fully described in detail in [2, 5]. This method enables simultaneous faults to be identified in terms of position and severity. An outline of this identification technique is shown below tailored to the specific experimental case, taking into consideration that in the experimental case presented the location of the fault is already known.

As said above, the dynamic effects of machine faults can be simulated by suitable sets of forces and moments that are applied to nodes of the FE model of the machine rotor train. By assuming that the system is linear and time invariant, which is rather acceptable in machines like that considered in the paper, the following equation can be written for each harmonic component, of order $n$, of the machine excitations:

$$
\left[-(n \Omega)^{2} \mathbf{M}+i n \Omega \mathbf{C}+\mathbf{K}\right] \mathbf{X}_{n}=\mathbf{F}_{n}(\Omega)
$$

where $\Omega$ is the machine rotating speed, $\mathbf{K}, \mathbf{M}$ and $\mathbf{C}$ are the stiffness, mass and damping (including gyroscopic effect) matrices of the fully assembled system composed of the rotor-train, bearings and foundation structure. The terms of the force vector $\mathbf{F}_{n}$ are the equivalent excitations used to model the fault that have to be identified:

$$
\mathbf{F}_{n}=\sum_{i=1}^{m} \mathbf{F}_{n}^{(i)}
$$

In the case considered in the paper, in which two (i.e. $m=2$ ) pairs of bending moments are going to be identified for the first harmonic component (i.e. $n=1$ ), the force vector for each pair $k$ of bending moments can be expressed as:

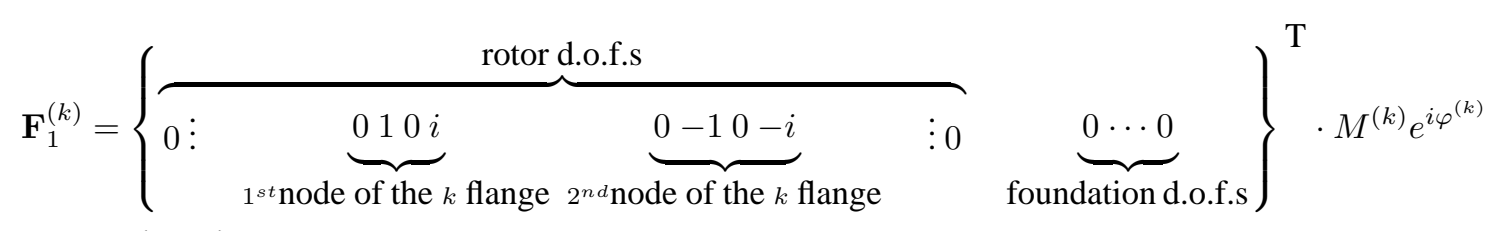

$$
\begin{aligned}
& =\left\{\mathbf{F}_{L}^{(k)}\right\} \bar{A}^{(k)}
\end{aligned}
$$

where the only elements different from zero are those relative to the horizontal and vertical rotational d.o.f.s of the element corresponding to the flange $k$ where the moments are applied. Equation (17) can be rewritten considering that condition monitoring systems collects data for many rotating speeds, so the additional vibrations are available for several rotating speeds and a set of $p$ rotating speeds is considered:

$$
\vec{\Omega}=\left\{\Omega_{1} \Omega_{2} \ldots \Omega_{n}\right\}^{T}
$$

The quantity in the square brackets of Eq. (17) is the dynamical stiffness matrix $\mathbf{E}$ of the system. Although Eq. (17) can be written for every rotating speed of a transient, only the experimental vibration data collected at $p$ rotating speeds are analysed in order to avoid an overabundance of nearly identical observations that could cause numerical problems or identification errors and that could cause the solution of the problem to become time consuming.

$$
[\mathbf{E}(n \vec{\Omega})] \mathbf{X}_{n}=\left[\begin{array}{cccc}
\mathbf{E}\left(n \Omega_{1}\right) & 0 & 0 & 0 \\
0 & \mathbf{E}\left(n \Omega_{2}\right) & 0 & 0 \\
\vdots & \vdots & \vdots & \vdots \\
0 & 0 & 0 & \mathbf{E}\left(n \Omega_{p}\right)
\end{array}\right]\left\{\begin{array}{c}
\mathbf{X}_{n} \\
\mathbf{X}_{n} \\
\vdots \\
\mathbf{X}_{n}
\end{array}\right\}=\left\{\begin{array}{c}
\sum_{i=1}^{m} \mathbf{F}_{f_{n}}^{(i)}\left(\Omega_{1}\right) \\
\sum_{i=1}^{m} \mathbf{F}_{f_{n}}^{(i)}\left(\Omega_{2}\right) \\
\vdots \\
\sum_{i=1}^{m} \mathbf{F}_{f_{n}}^{(i)}\left(\Omega_{p}\right)
\end{array}\right\}=\mathbf{F}_{f_{n}}(\vec{\Omega})
$$

Some criteria used to select the rotating speeds at which significant transient vibrations can be considered are described in [31].

The effect on the measured d.o.f.s $\hat{\mathbf{X}}_{\mathrm{B}_{1}}$, due to unitary bending moments applied to the elements of the model corresponding to the flanges, is now calculated. This is done by inverting matrix $\mathbf{E}(n \Omega)$ and obtaining the transfer matrix.

$$
\mathbf{X}_{n}=[\mathbf{E}(n \vec{\Omega})]^{-1} \mathbf{F}_{n}(\vec{\Omega})=[\mathbf{H}(n \vec{\Omega})] \mathbf{F}_{n}(\vec{\Omega})
$$


Then, the vibrations of the d.o.f.s, which are measured, are separated from the all the d.o.f.s of the system, by considering only the rows of $\mathbf{H}(n \vec{\Omega})$ corresponding to the measured d.o.f.s. Also the weight matrix, which is for the $j$-th rotating speed:

$$
\left[\mathbf{W}^{(j)}\right]=\operatorname{diag}\left(w_{1}^{(j)}, \ldots, w_{21}^{(j)}\right)
$$

and where $w_{i}^{(j)}=1$ indicates that the corresponding measure is fully considered at the $j$-th rotating speed, is introduced and results:

$$
\left[\hat{\mathbf{X}}_{\mathrm{B}_{1}}\right]=\left.[\mathbf{W}][\mathbf{H}(n \vec{\Omega})]\right|_{\substack{\text { measured } \\
\text { d.o.f.s }}}\left[\begin{array}{lll}
\mathbf{F}_{L}^{(1)} & \vdots & \mathbf{F}_{L}^{(m)}
\end{array}\right]
$$

Now the array of the complex values $\bar{A}^{(k)}$ (i.e. the modules and phases) of the equivalent force systems applied in the first node that fits best the experimental data $\mathbf{X}_{\mathbf{B} m_{1}}$, have to be estimated. The fitting is done in least square sense, since the number of the unknown (the modules and the phases) is less than the equations (recalling that data are corresponding to several rotating speeds and each of the sets is composed by several measuring planes, while the number of the faults is two in this case). The problem is equivalent to:

$$
\min \left\|\left[\hat{\mathbf{X}}_{\mathrm{B}_{1}}\right]\left\{\begin{array}{c}
A^{(1)} \\
\vdots \\
A^{(m)}
\end{array}\right\}-\mathbf{X}_{\mathrm{B} m_{1}}\right\|
$$

whose general solution is given by the pseudo-inverse calculation:

$$
\mathbf{A}=\left(\left[\hat{\mathbf{X}}_{\mathrm{B}_{1}}\right]^{\mathrm{T}}\left[\hat{\mathbf{X}}_{\mathrm{B}_{1}}\right]\right)^{-1}\left[\hat{\mathbf{X}}_{\mathrm{B}_{1}}\right]^{\mathrm{T}}[\mathbf{W}] \mathbf{X}_{\mathrm{B} m_{1}}
$$

A full discussion on the possible numerical errors in calculating Eq. (26) is reported in [1]. The modules and the phases of the complex values in the $m$ rows of $\mathbf{A}$ are the identified faults. Finally the residual is determined, first obtaining the calculated response due to the two identified pairs of bending moments:

$$
\mathbf{X}_{\mathrm{B}_{1}}=\left[\hat{\mathbf{X}}_{\mathrm{B}_{1}}\right] \mathbf{A}
$$

and then normalizing its difference with respect to the measured vibrations, obtaining the residual:

$$
\varepsilon=\left(\begin{array}{c}
{\left[\mathbf{X}_{\mathrm{B}_{1}}-\mathbf{X}_{\mathrm{B} m_{1}}\right]^{* \mathrm{~T}}\left[\mathbf{X}_{\mathrm{B}_{1}}-\mathbf{X}_{\mathrm{B} m_{1}}\right]} \\
\mathbf{X}_{\mathrm{B} m_{1}}^{* \mathrm{~T}} \\
\mathbf{X}_{\mathrm{B} m_{1}}
\end{array}\right)^{1 / 2}
$$

The closer to zero the value of Eq. (28) is, the better the estimation of the faults is.

\section{Case history}

A fault occurred in a small $50 \mathrm{MW}$ power plant has been analysed. The machine rotor-train was composed of a gas turbine, a generator and a steam turbine. The two turbines were mounted at the opposite ends of the generator. Two gearboxes were assembled to the machine train between the turbines and the generator. The machine train diagram is shown in Fig. 2. Shaft vibrations were measured with radial proximity probes mounted along two orthogonal directions $(X Y)$ in all the machine main bearings.

In order to limit the effects induced by torque changes and misalignments on the machine dynamic behaviour, the wheels of the gearboxes were mounted on quill-shafts. In addition, two flexible couplings were used to joint the gear shafts with generator and steam turbine, respectively. These transmission units were composed of a short hollow shaft at whose ends two flanges, linked to a membrane unit, were located (Fig. 3). Owing to the flexibility of these coupling units, partial angular, axial and radial deflections of the shafts joined to the flexible couplings were tolerated. 


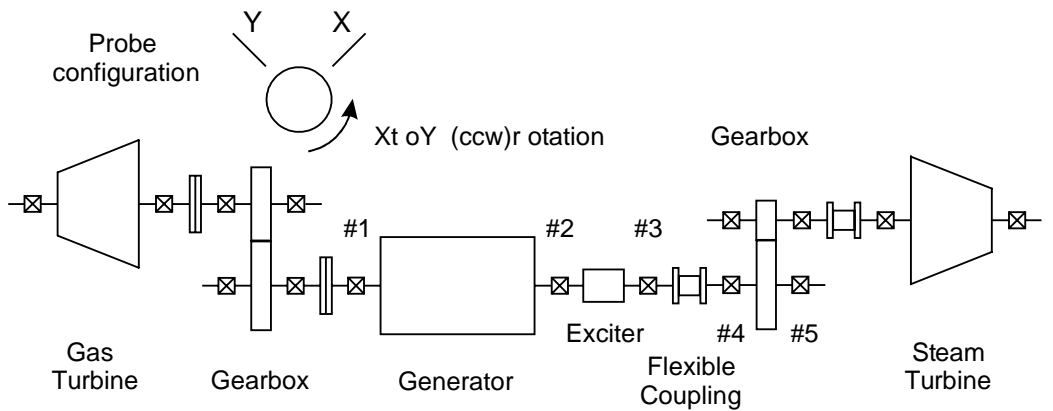

Fig. 2. Machine train diagram.

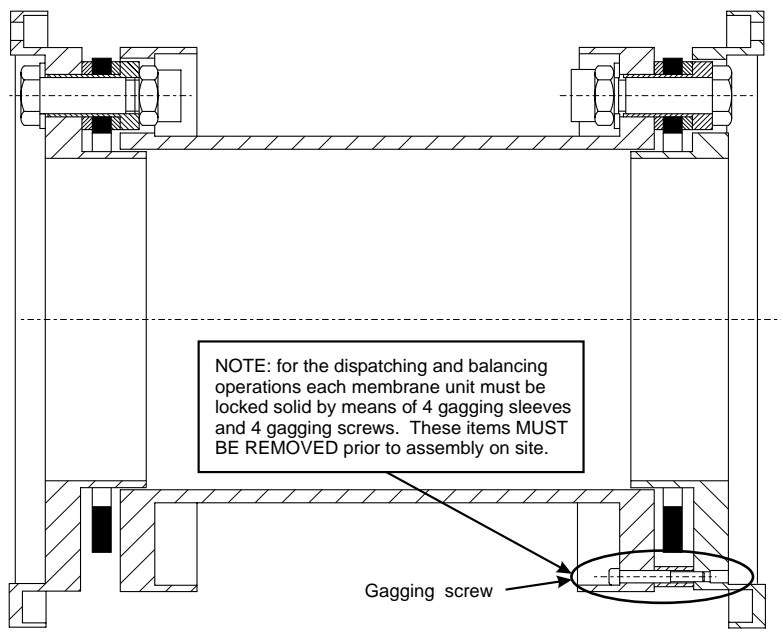

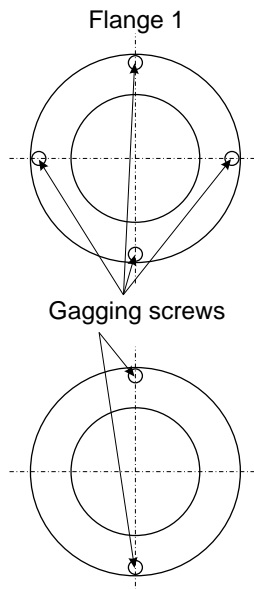

Flange 2

Fig. 3. Flexible coupling mounted between the generator and the slow shaft of the gearbox. Location of the gagging screws mounted on the flanges of the flexible coupling.

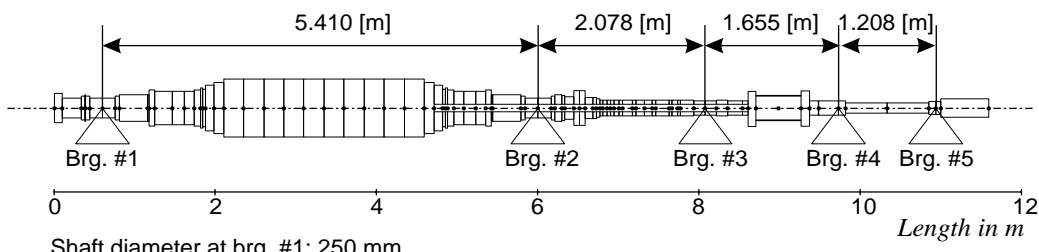

. Shaft diameter at brg. \#2: $250 \mathrm{~mm}$ Shaft diameter at brg. \#3: $190 \mathrm{~mm}$ Shaft diameter at brg. \#4: $190 \mathrm{~mm}$ Shaft diameter at brg. \#5: $160 \mathrm{~mm}$

Fig. 4. Draft of the rotor-train: generator (between brg. \#1 and \#2), exciter (between brg. \#2 and \#3), flexible coupling (between brg. \#3 and \#4), slow shaft of the geabox (between brg. \#4 and \#5).

In the following only the coupling between the generator and the steam turbine will be considered. The three journal bearings of the generator as well as the two main bearings of the slow shaft of the gearbox coupled with the steam turbine have been numbered from \#1 to \#5 as shown in Fig. 4.

Some important maintenance actions carried out on the rotor windings of the exciter required a long machine outage. During the maintenance the rotor-train was disassembled and some bearings were dismounted. These important overhauls caused the flexible couplings to be removed.

When the maintenance actions were completed the machine train was reassembled and aligned. Sophisticated laser sensors were used to check both angular and radial misalignment between generator and gearbox. During these 
Table 1

\begin{tabular}{|c|c|c|c|c|}
\hline Bearing $\mathrm{n}^{\circ}$ & SDI & $\begin{array}{c}\text { Major axis inclination } \\
\text { degrees }\end{array}$ & $\begin{array}{c}\text { Major axis amplitude } \\
\mu \mathrm{m}\end{array}$ & $\begin{array}{c}\text { Minor axis amplitude } \\
\qquad \mu \mathrm{m}\end{array}$ \\
\hline$\# 1$ & 0.0184 & $41^{\circ}$ & 7.1 & 0.1 \\
\hline \#2 & 0.0351 & $8^{\circ}$ & 25.0 & 0.9 \\
\hline \#3 & 0.1362 & $-16^{\circ}$ & 54.4 & 7.4 \\
\hline \#4 & 0.3275 & $83^{\circ}$ & 72.2 & 23.6 \\
\hline
\end{tabular}

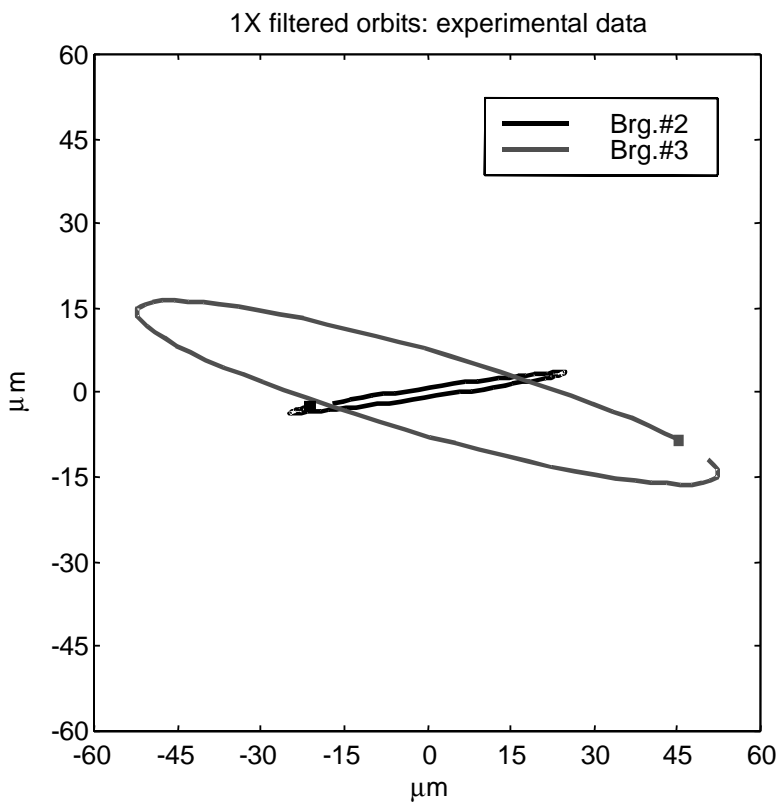

Fig. 5. 1X filtered orbrits in bearings \#2 and \#3.

maintenance actions the flexible couplings were not assembled to the rotor-train. Only when the machine alignment was completed and checked, the generator rotor and the slow shaft of the gearbox were jointed by means of the flexible transmission unit shown in Fig. 3.

Before doing a first complete runup, the machine composed of the gas turbine and the generator was started-up and the rotating speed of the generator was increased to $150 \mathrm{rpm}$. In this operating condition the shaft vibrations at some measurement points showed to be quite abnormal. The maximum amplitudes of the synchronous vibrations (1X) measured on bearings \#2 and \#3 reached $50 \mu \mathrm{m}$ pk-pk and $109 \mu \mathrm{m}$ pk-pk, respectively (Fig. 5). On bearing \#4, mounted on the slow shaft of the gearbox, the amplitude of the $1 \mathrm{X}$ vibrations reached $144 \mu \mathrm{m}$ pk-pk (Fig. 6). These vibration levels are noticeable if we consider that the shaft rotating speed was very low. Therefore, the runup was aborted immediately to avoid possible severe damages.

In order to obtain significant diagnostic information the machine synchronous vibrations have been used to evaluate the 1X filtered orbits at the support of the generator and the slow shaft of the gearbox. The major and minor axes of the elliptic orbits have been evaluated along with the SDI and the inclination angle of the major axis with respect to the horizontal direction. Table 1 shows the results obtained by the analysis of the raw $1 \mathrm{X}$ vibrations measured at $150 \mathrm{rpm}$ during the first aborted runup.

Also the analysis of the $2 \mathrm{X}$ vibrations measured at $150 \mathrm{rpm}$ highlighted abnormal vibration levels especially for the bearings \#3 and \#4, as reported in Table 2.

It was quite evident that the machine was affected by a serious failure caused by a wrong machine reassembling. Owing to the low value of the rotating speed it was unlikely that the machine vibrations could be caused by a shaft unbalance. The low SDI values indicate that the degree of ellipticity of the $1 \mathrm{X}$ orbits determined on the generator 
Table 2

\begin{tabular}{|c|c|c|}
\hline Bearing $\mathrm{n}^{\circ}$ & $\begin{array}{l}\mathrm{X} \text { direction } \\
\mu \mathrm{m} \text { pk-pk }\end{array}$ & $\begin{array}{l}\text { Y direction } \\
\mu \mathrm{m} \text { pk-pk }\end{array}$ \\
\hline$\# 1$ & 9.0 & 3.9 \\
\hline$\# 2$ & 6.1 & 8.7 \\
\hline \#3 & 24.3 & 27.8 \\
\hline$\# 4$ & 26.0 & 29.6 \\
\hline
\end{tabular}

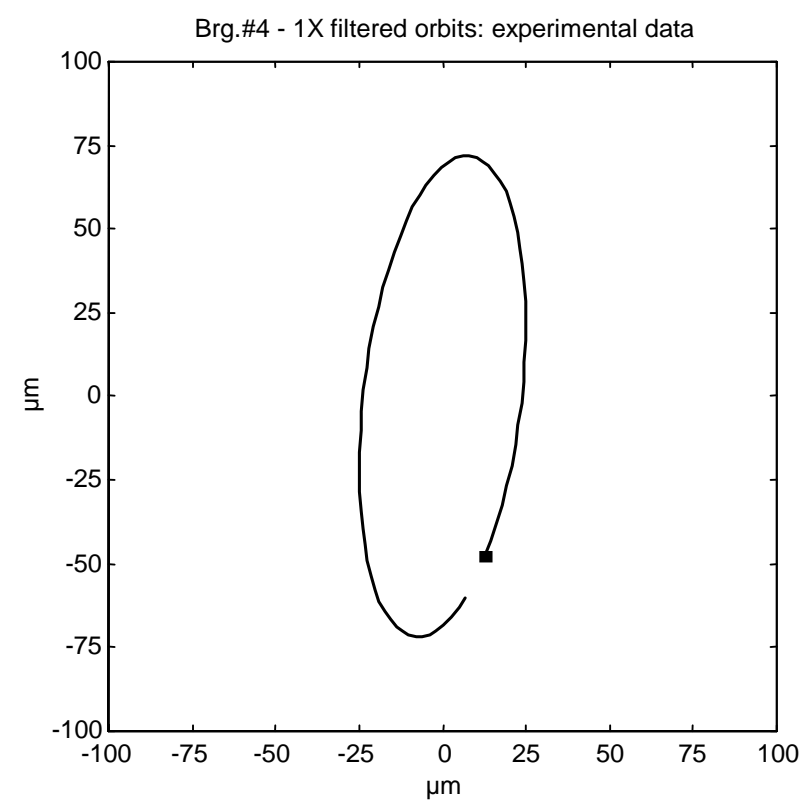

Fig. 6. $1 \mathrm{X}$ filtered orbits in bearing \#4.

journal bearings \#2 and \#3 was noticeable. The collapse of the $1 \mathrm{X}$ orbits towards a straight line in case of severe angular misalignment is also observed in [18], even in a test-rig equipped by ball bearings. This effect is more evident in case of less stiff bearing like those of the considered machine that are oil-film journal bearings. At low rotating speeds the oil-film stiffness of the journal bearings \#1, \#2 and \#3 was very high in the vertical direction but rather weak in the horizontal direction: this made the effects of the fault more serious. Moreover, on these two bearings, the shaft vibrations were mainly horizontal while the vertical vibrations were nearly null (Table 1). Conversely, the inclination angle of the major axis of the elliptical orbit measured on bearing \#4 shows that the maximum $1 \mathrm{X}$ vibrations at this support occurred nearly in vertical direction.

The keyphasor dot of the $1 \mathrm{X}$ filtered orbits evaluated on bearings \#2 and \#3 showed that the phases of the dynamic deflection of the shaft on these two adjacent supports were nearly opposite. This additional symptom suggested to suppose that the machine failure was able to induce a shaft bending in the machine train. Also the presence of a significant $2 \mathrm{X}$ vibration component is a symptom of coupling misalignment $[25,26,28]$. Therefore, the most probable fault was a severe misalignment which affected the coupling between the generator and the steam turbine. This was surprising because a great care had been taken to align this part of the rotor-train. Vibration measurements carried out at $150 \mathrm{rpm}$ during further aborted runups confirmed that this abnormal behaviour repeated itself showing the same fault symptoms.

A machine visual inspection showed that in the two flanges located at the opposite ends of the flexible coupling six gagging sleeves and gagging screws were mounted in the diametrical opposite locations showed in Fig. 3. A note written on a technical drawing of the transmission unit explains that these screws can be used to lock solid the membrane units for the dispatching and balancing operations. The same note, reported also in Fig. 3, emphasises that the screws must be removed prior to assembly the transmission unit on site. Moreover, they had been tightened 
Table 3

Parameters of the $1 \mathrm{X}$ filtered orbits caused by the machine residual unbalance. Analysis of experimental vibrations

$\begin{array}{crccc}\text { Bearing } \mathrm{n}^{\circ} & \text { SDI } & \begin{array}{c}\text { Major axis inclination } \\ \text { degrees }\end{array} & \begin{array}{c}\text { Major axis amplitude } \\ \mu \mathrm{m}\end{array} & \begin{array}{c}\text { Minor axis amplitude } \\ \# 1\end{array} \\ & 0.7289 & 109^{\circ} & 4.7 & \mu \mathrm{m} \\ \# 2 & -0.0153 & 15^{\circ} & 6.8 & 3.5 \\ \# 3 & 0.7463 & 68^{\circ} & 8.8 & 0.2 \\ \# 4 & 0.2252 & 120^{\circ} & 5.1 & 6.5 \\ \end{array}$

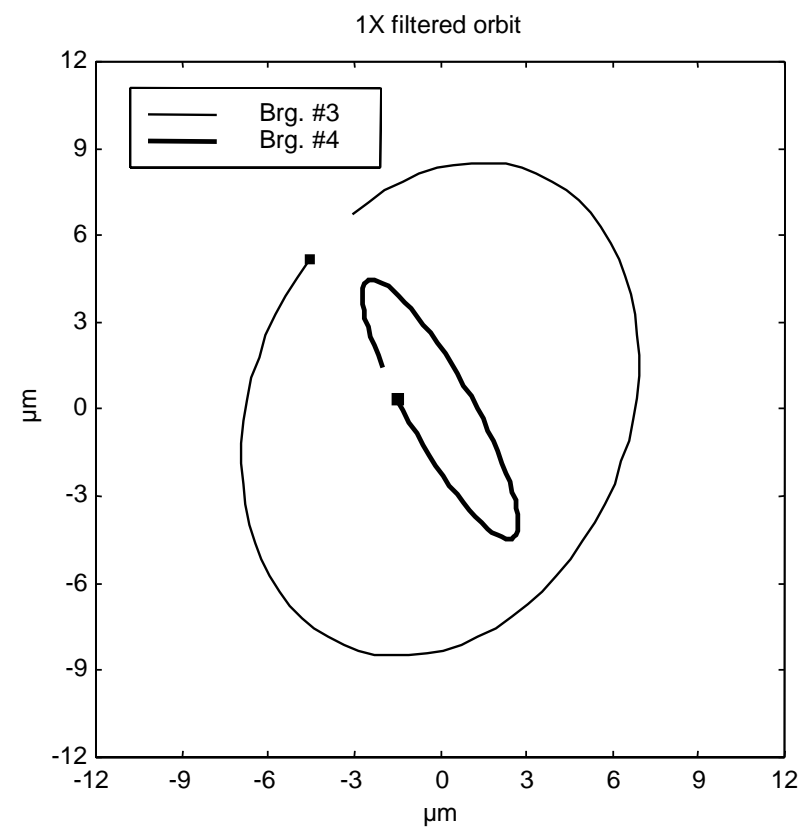

Fig. 7. 1X filtered orbit induced by the residual unbalance of the machine on bearings \#3 (gererator) and \#4 (gearbox). Experimental data.

heavily and, likely, not homogeneously. Owing to this an angular deflection was induced at the ends of the flexible coupling. The gagging screws were never removed from the flanges. When this transmission unit was assembled to the machine rotor-train an undesired angular misalignment was induced in the coupling between the generator and the gearbox.

After having removed the gagging screws mounted erroneously on the flexible coupling the power unit was started-up again. The machine vibrations at the rotating speed of $150 \mathrm{rpm}$ were measured and analysed. In this case the maximum amplitude of the shaft vibrations on the bearings from \#1 to \#4 did not exceed $9 \mu \mathrm{m}$ pk-pk.

Table 3 shows the parameters of the $1 \mathrm{X}$ filtered orbits occurred at $150 \mathrm{rpm}$ after having removed the gagging screws from the flexible coupling. Figure 7 shows the orbits determined on bearings \#3 and \#4 in this normal operating condition. On bearing \#3, that is very close to the exciter and the coupling, the orbit associated with the reference $1 \mathrm{X}$ vibrations was nearly circular $(\mathrm{SDI}=0.7463)$. On the contrary, before removing the gagging screws that locked solid the membrane unit the orbit on this support was highly elliptical $(\mathrm{SDI}=0.1362)$.

These vibration levels were sufficiently low and did not exceed the respective reference values obtained by the analysis of the transient vibrations collected during previous runups occurred before the machine maintenance. The $2 \mathrm{X}$ are also sensibly reduced, as can be noticed by comparing the raw data reported in Table 4 to those of Table 2 .

Therefore, it was confirmed that the high abnormal vibrations occurred during the first aborted runup were caused by a shaft bending induced by the screws mounted erroneously on the flexible coupling. 
Table 4

Raw 2X filtered vibrations measured at $150 \mathrm{rpm}$ having removed the gagging screws from the flexible coupling

$\begin{array}{ccc}\text { Bearing } \mathrm{n}^{\circ} & \begin{array}{c}\mathrm{X} \text { direction } \\ \mu \mathrm{m} \text { pk-pk }\end{array} & \begin{array}{c}\mathrm{Y} \text { direction } \\ \mu \mathrm{m} \text { pk-pk }\end{array} \\ \# 1 & 9.4 & 3.9 \\ \# 2 & 2.7 & 5.7 \\ \# 3 & 5.3 & 1.5 \\ \# 4 & 11.1 & 11.0\end{array}$

Table 5

Identified opposite bending moments applied to the flanges of the flexible coupling unit (see Fig. 8)

Location

Identified bending moments

Flange 1 $\left(M_{1}\right) \quad \begin{array}{r}\text { Amplitude } \\ 1906 \mathrm{Nm}\end{array}$

Phase A $(\varphi) \quad$ Phase B $\left(\varphi+180^{\circ}\right)$

Flange $2\left(M_{2}\right) \quad 1653 \mathrm{Nm}$ $93^{\circ}$

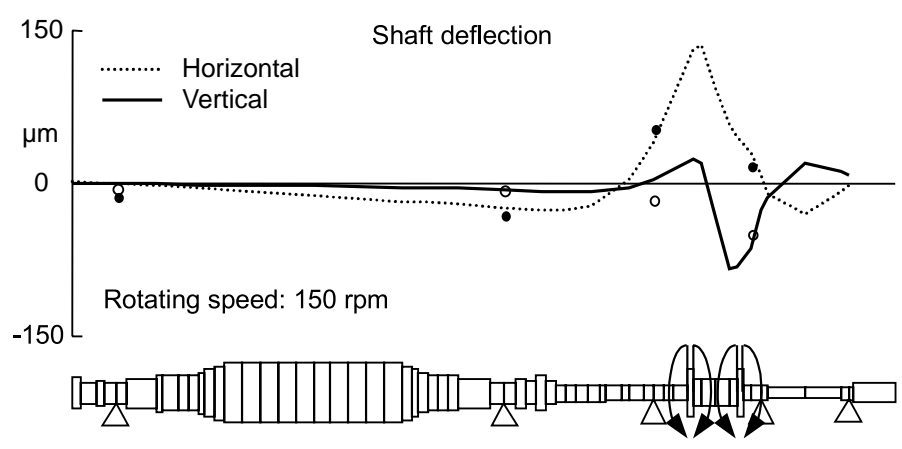

Fig. 8. Dynamic defelction of the rotor-train induced at $150 \mathrm{rpm}$ by the identified bending moments $M_{1}$ and $M_{2}$. Solid dots: experimental deflection in horizontal direction. Circles: experimental deflections in vertical direction.

\section{Fault identification}

A model of the machine train composed of the generator, the flexible coupling and the driven shaft of the gear has been used to identify the bending moments caused by the gagging screws that have been mounted on the coupling. The model is composed of 85 beam elements, as shown in Fig. 4, while linearized damping and stiffness coefficient depending on the rotating speed have been used for the 5 bearings. The supporting structure is modelled by means of pedestals. The model considers the gyroscopic, the shear and the secondary inertia effects. The effects of the screw tightening can be simulated by applying pairs of opposite bending moments to suitable nodes of the finite element model of the rotor-train. In this case, the axial positions of the moments along the machine were known. Conversely, the angular position and the amplitude of these moments have been estimated by means of a model-based identification method [2-5].

Two pairs of opposite bending moments have been considered. Each pair of moments has been applied to the ending nodes of the beam finite element with which each flange of the flexible coupling has been modelled (Fig. 8).

The $1 \mathrm{X}$ vibrations measured at $150 \mathrm{rpm}$ after having removed the screws have been used as reference vectors. These vectors have been subtracted from the corresponding abnormal vibrations vectors measured during the first runup. In this way, the $1 \mathrm{X}$ vibrations induced only by the fault, that is by the shaft bending, were estimated. Hereafter these vibrations will be called additional vibrations. Figure 9 illustrates the $1 \mathrm{X}$ filtered orbits determined using the additional vibrations evaluated at $150 \mathrm{rpm}$ on bearings \#3 and \#4. Owing to the low amplitude of the $1 \mathrm{X}$ reference vibrations the parameters of these orbits, here not reported for the sake of brevity, were very similar to those shown in Table 1. 
Table 6

Parameters of the $1 \mathrm{X}$ filtered orbits caused by the identified bending moments. Analysis of simulated vibrations

\begin{tabular}{ccccc} 
Bearing $\mathrm{n}^{\circ}$ & SDI & $\begin{array}{c}\text { Major axis inclination } \\
\text { degrees }\end{array}$ & $\begin{array}{c}\text { Major axis amplitude } \\
\mu \mathrm{m}\end{array}$ & $\begin{array}{c}\text { Minor axis amplitude } \\
\text { \#1 }\end{array}$ \\
\hline & 0.1299 & $5^{\circ}$ & 1.3 & $\mu \mathrm{m}$ \\
$\# 2$ & 0.0777 & $14^{\circ}$ & 26.3 & 0.2 \\
$\# 3$ & 0.1144 & $1^{\circ}$ & 43.7 & 2.3 \\
$\# 4$ & 0.3599 & $94^{\circ}$ & 77.6 & 5.7
\end{tabular}
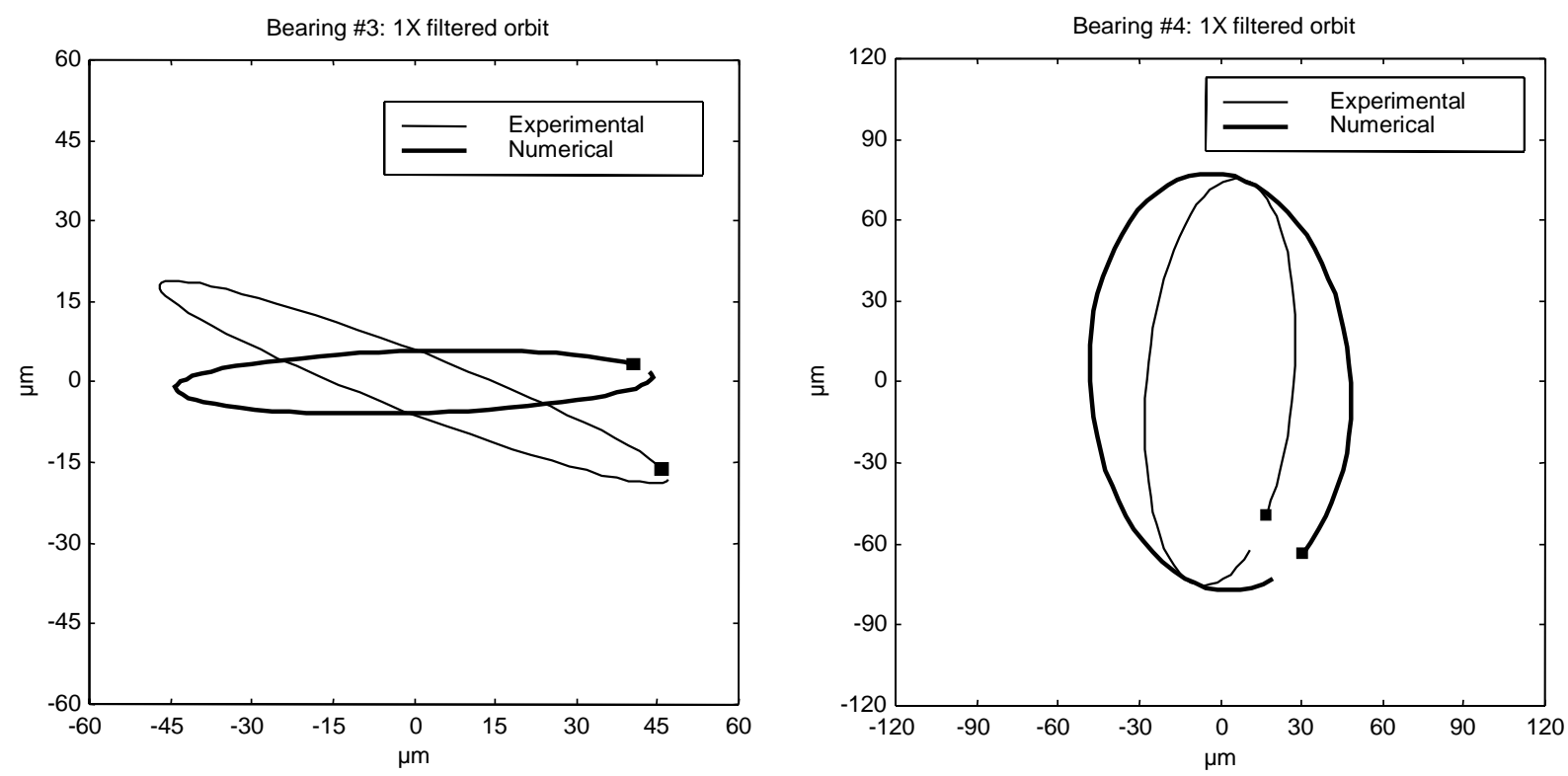

Fig. 9. 1X filtered orbit induced by the fault on bearing \#3 and \#4 (generator). Comparison between experimental data and numerical results.

The additional vibration data, that is the experimental vibrations that can be considered the consequence of the fault only, have been processed with the Weighted Least Squares Error method presented before in order to identify the bending moments. Table 5 shows the amplitude and the phase of the identified bending moments. The residual $\varepsilon$ obtained with this case study was 0.1292 . That is the relative error of the identification process, evaluated with Eq. (28), was rather low although the effects of the significant anisotropy of the oil-film stiffness of the generator bearings were difficult to be simulated with a high accuracy.

The 1X elliptical orbits induced at $150 \mathrm{rpm}$ by the identified bending moments have been evaluated. The journal orbits simulated at bearings \#3 and \#4 are shown in Fig. 9 together with the respective experimental orbits. The amplitude of the major and minor axes of the $1 \mathrm{X}$ filtered orbits, as well as the degree of ellipticity of each orbit, are shown in Table 1. These results can be compared with those reported in Table 6. These accordance between the experimental orbits and those provided by the simulating model, in which the identified moments have been considered, is satisfactory.

It is necessary to consider that, in this case, it is rather difficult to simulate the high degree of ellipticity of the experimental orbits. Nevertheless, the simulated orbits on bearings \#2 and \#3 are rather flat, their major axis is nearly horizontal and the keyphasor dots are in opposite angular positions in accordance with the experimental orbits. Also the maximum amplitude of the $1 \mathrm{X}$ experimental vibrations on the bearing \#4 mounted on the slow shaft of the gearbox is well fitted by the numerical response.

The machine train deflection induced at $150 \mathrm{rpm}$ by the identified bending moments has been evaluated. Figure 8 shows the shape of the horizontal and vertical components of this dynamic deflection along the generator and the slow shaft of the gearbox. In the lower part of Fig. 8 the finite element model of the rotor-train is shown along with the location of the two pairs of opposite bending moments, $M_{1}$ and $M_{2}$, that have been identified. In the same figure 

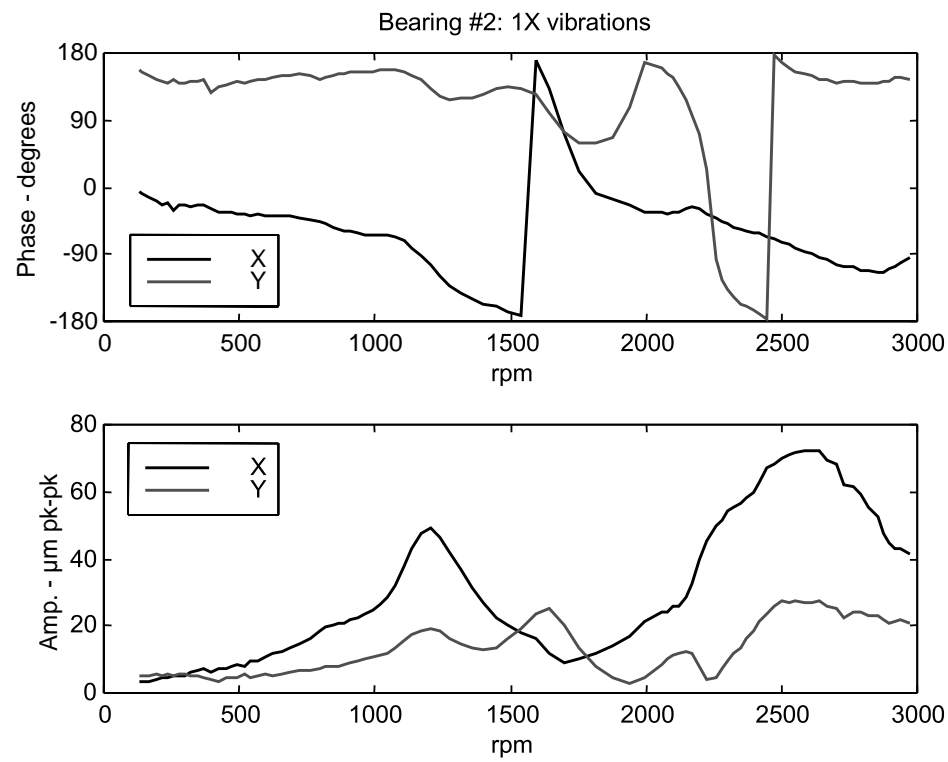

Fig. 10. Bode plot of the $1 \mathrm{X}$ experimental transient vibrations on bearing \#2. These vibrations have been assumed as reference data $\mathbf{X}_{\mathrm{ef}}$. Machine trip level is $180 \mu \mathrm{m}$ pk-pk.

the experimental values of the deflections measured at $150 \mathrm{rpm}$ at the bearings numbered from \#1 to \#4 are shown. Also in this case the accordance between experimental data and numerical results is satisfactory. The maximum deflection of the shaft reached a predicted amplitude of nearly $280 \mu \mathrm{m}$ pk-pk in a cross-section of the shaft fairly close to a measurement point. Owing to the angular misalignment induced by the flexible coupling the rotor-train was affected by a bend that caused deflections with opposite phases on the generator bearings \#2 and \#3.

\section{Prediction of the machine dynamical behaviour}

After having removed the gagging screws from the transmission unit, the machine was started-up and the rotating speed reached the machine operating speed $(3000 \mathrm{rpm})$. In all the machine measurement points the amplitude of the $1 \mathrm{X}$ transient vibrations did not exceed the alarm limit value that was set to $180 \mu \mathrm{m}$ pk-pk. On the contrary, these transient vibrations showed to be sufficiently low. In the following, the $1 \mathrm{X}$ vibrations measured during this runup have been considered as reference vectors and indicated as $\mathbf{X}_{r e f}$. Figure 10 shows the Bode plot of the $1 \mathrm{X}$ vibrations measured on bearing \#2 during the runup.

The model of the fully assembled machine and the two pairs of bending moments, previously identified, have been used to predict the $1 \mathrm{X}$ transient vibrations $\overline{\mathrm{X}}$ that would have occurred if the first runup had not been aborted at an early stage. In order to estimate the $1 \mathrm{X}$ vibrations $\overline{\mathrm{X}}$ that would have been caused by the concomitant effects of the residual unbalance and the angular misalignment of the flexible coupling the experimental $1 \mathrm{X}$ vibration vectors $\mathbf{X}$ ref measured during the reference transient have been added to the corresponding $1 \mathrm{X}$ vectors $\mathbf{X}_{1}$ provided by the model of the fully assembled machine in which the identified bending moments have been used as machine excitation:

$$
\overline{\mathbf{X}}=\mathbf{X}_{\text {ref }}+\mathbf{X}_{1}
$$

Using the values of the identified bending moments, provided in Table 5, Eq. (19) becomes:

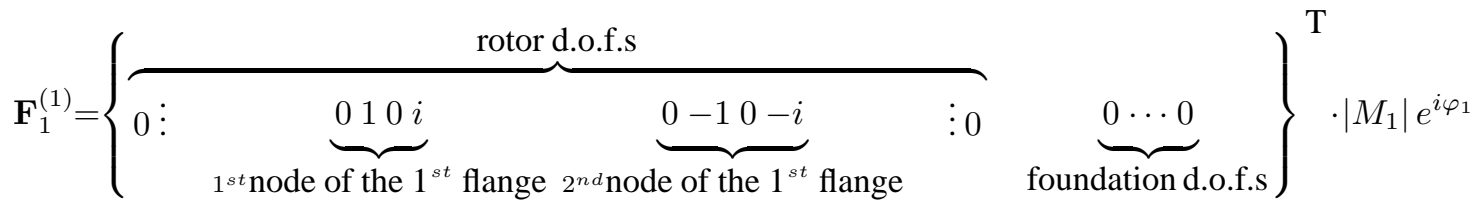



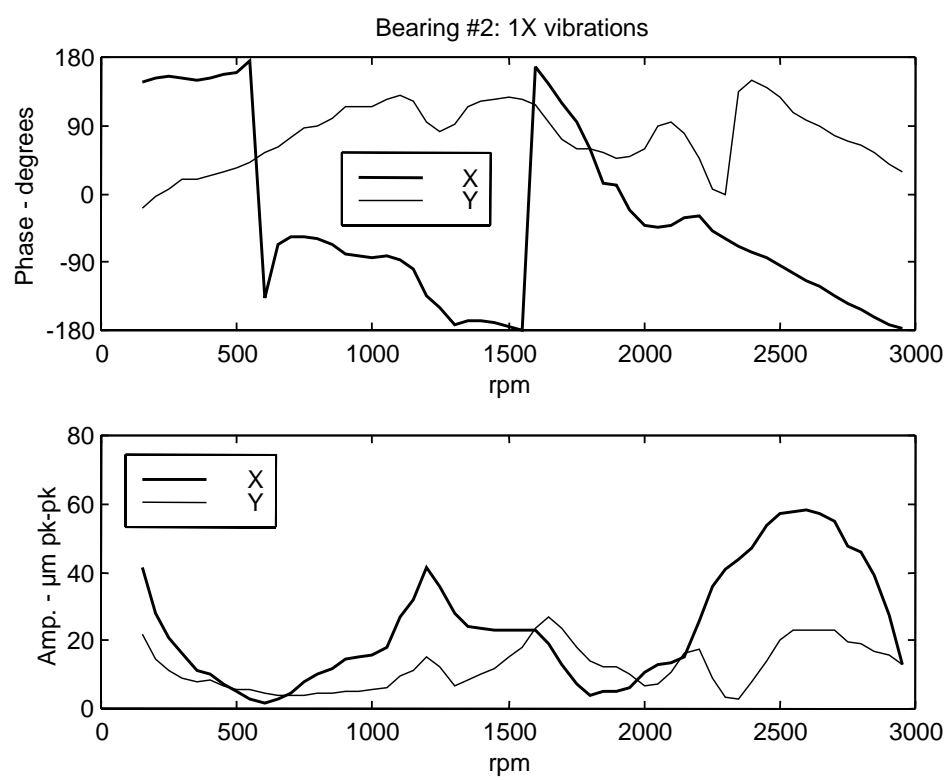

Fig. 11. Bode plot of the $1 \mathrm{X}$ transient vibrations $\mathbf{X}=\mathbf{X}_{\mathrm{ref}}+\mathbf{X}_{1}$ induced on bearing \#2 by the identified bending moments and the machine residual unbalance. Vibrations estimated by means of the simulating model. Machine trip level is $180 \mu \mathrm{m}$ pk-pk.

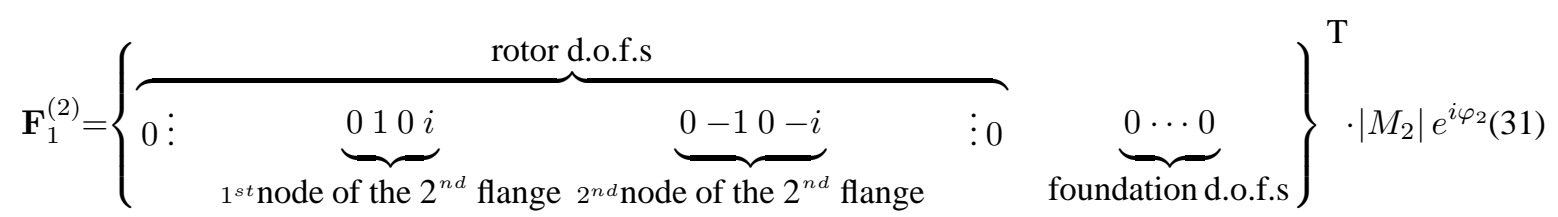

Equations (30) and (31) are substituted first in Eq. (18), then in the 1.h.s of Eq. (22) and the vibrations $\mathbf{X}_{1}$ due to the identified bending moments are calculated.

Figure 11 shows the Bode plot of the $1 \mathrm{X}$ transient vibrations $\overline{\mathbf{X}}$ evaluated in this way at the generator bearing \#2.

On the basis of the results obtained with this investigation the amplitude of the $1 \mathrm{X}$ vibrations on bearing \#2 would have not exceeded the alarm limit values over the complete speed range up to $3000 \mathrm{rpm}$. Being confident that the vibrations predicted with the model are quite reliable, the machine could be started-up also with the gagging screws mounted on the flexible coupling. However, although the $1 \mathrm{X}$ vibration levels would have not exceeded the danger limits (machine trip level is $180 \mu \mathrm{m}$ pk-pk), both at the operating speed and in the balance resonance region, the machine dynamic behaviour would have been critical, especially at low rotating speeds. In addition, the flexible coupling would have not operated correctly and, likely, it would have been affected by abnormal stresses.

However, if it had been necessary to operate the machine on load at any rate, for a not too long time, this prediction of the shaft vibrations would have allowed to be more confident on the possibility of carrying out a complete runup without causing a catastrophic damage.

\section{Conclusions}

The orbit shape analysis has allowed an angular misalignment in a generator of a small power plant to be identified. Then, a model-based identification method has been used to estimate the bending moments that have enabled the $1 \mathrm{X}$ vibrations caused by the angular misalignment to be simulated. This investigation, as well as a machine visual inspection, proved that the high $1 \mathrm{X}$ vibrations that occurred at low rotating speeds were caused by a misalignment induced by the unsuitable mounting of gagging screws that locked solid the membrane unit of a flexible coupling between two shafts of the machine train.

The identified bending moments allowed the experimental vibration data collected at low rotating speeds to be fitted successfully. Moreover, a simulating model and the identified bending moments have been used to estimate the 
dynamic deflection of the machine train induced at low rotating speeds by the angular misalignment. This diagnostic information proved to be useful to evaluate the severity of the effects caused by the machine misalignment.

In addition to this, the identified bending moments have been used to predict the $1 \mathrm{X}$ machine vibrations that would have occurred if the first runup had not been aborted. This investigation has provided further interesting diagnostic information.

The diagnostic methods used in the investigations described in this paper have shown to be very useful and effective to satisfy some basic requirements of the fault diagnosis and prognosis in rotating machines. The successful results provided by the case study shown in the paper prove that usual condition monitoring techniques can be combined and integrated with model-based methods to enable a complete machine health analysis as well as to provide some important information required by protective and predictive maintenance.

\section{References}

[1] E. Anderson, Z. Bai, C. Bischof, S. Blackford, J. Demmel, J. Dongarra, J. Du Croz, A. Greenbaum, S. Hammarling, A. McKenney and D. Sorensen, LAPACK User's Guide, 3rd Edition, SIAM, Philadelphia, 1999.

[2] N. Bachschmid and P. Pennacchi, Multiple fault identification method in the frequency domain for rotor systems, Shock and Vibration 9 (2002), 203-215.

[3] N. Bachschmid and P. Pennacchi, Accuracy of fault detection in real rotating machinery using model based diagnostic techniques, JSME International Journal Series C 46 (2003), 1026-1034.

[4] N. Bachschmid, P. Pennacchi, E. Tanzi and A. Vania, Accuracy of Modelling and Identification of Malfunctions in Rotor Systems: Experimental Results, Journal of Brazilian Society of Mechanical Sciences XXII (2000), 423-442.

[5] N. Bachschmid, P. Pennacchi and A. Vania, Identification of Multiple Faults in Rotor Systems, Journal of Sound and Vibration 254 (2002), $327-366$.

[6] N. Bachschmid, P. Pennacchi and A. Vania, Diagnostic Significance of Orbit Shape Analysis and its Application to Improve Machine Faults Detection, in printing on Journal of the Brazilian Society of Mechanical Sciences 1-32.

[7] P. Bonello and M.J. Brennan, Modelling the dynamic behaviour of a supercritical rotor on a flexible foundation using the mechanical impedance technique, Journal of Sound and Vibration 239 (2001), 445-466.

[8] N. Feng and E.J. Hahn, Identification of pedestal parameters in rotor-bearing-pedestal systems using known unbalance, ASME paper DETC2001/VIB-21553, 2001, Pittsburgh, Pennsylvania, 1-9.

[9] N. Feng and E.J. Hahn, Numerical evaluation of an identification technique for flexibly supported rigid turbomachinery foundations, Proc. of IFToMM- $6^{\text {th }}$ International Conference on Rotor Dynamics, 2002, Sydney (Australia), 854-861.

[10] W. Hu, H. Miah, N.S. Feng and E.J. Hahn, A Rig for Testing Lateral Misalignment Effects in a Flexible Rotor Supported on Three or More Hydrodynamic Journal Bearings, Tribology International 33 (2000), 197-204.

[11] R. Isermann, Fault detection and diagnosis - Methods and applications, Proc. of $2^{\text {nd }}$ International Symposium on Acoustical and Vibratory Surveillance Methods and Diagnostic Techniques, 1995, Senlis (France), 777-797.

[12] R. Isermann, Supervision, fault-detection and fault-diagnosis methods - An introduction, Control Engineering Practice 5 (1997), 639-652.

[13] R. Isermann and P. Ballé, Trends in the Application of Model-based Fault Detection and Diagnosis of Technical Process, Control Engineering Practice 5 (1997), 709-719.

[14] J. Kiciński, Model-based diagnostics in 200MW turbosets, Proc. of the $4^{\text {th }}$ Int. Conference on Acoustical and Vibratory Surveillance Methods and Diagnostic Techniques, 2001, Compiègne (France), 365-377.

[15] M. Lalanne and G. Ferraris, Rotordynamics Prediction in Engineering, John Wiley \& Sons Inc, Chichester, England, 1998.

[16] C.W. Lee, Directional Time-Frequency Signal Processing Techniques for Transient Rotating Machine Vibration Analysis, Proc. of the 10h World Congress of the Theory of Machines and Mechanisms, 1999, Oulu (Finland), 1692-1697.

[17] C.W. Lee and Y.-S. Han, The Directional Wigner Distribution and its Applications, Journal of Sound and Vibration 216 (1998), 585-600.

[18] Y.-S.Lee and C.-W. Lee, Modelling and Vibration Analysis of Misaligned Rotor-Ball Bearing Systems, Journal of Sound and Vibration 224 (1999), 17-32.

[19] R. Markert, R. Platz and M. Seidler, Model Based Fault Identification in Rotor Systems by Least Squares Fitting, Proc. of ISROMAC-8, Vol. II, 2000, Honolulu (Hawaii), 901-907.

[20] I. Mayes and J.E.T. Penny, Model based diagnostics of fault in rotating machines, Proc. of 12th International Congress on Condition Monitoring and Diagnostic Engineering Management - COMADEM 99, 1999, Sunderland (UK), 431-440.

[21] Z. Peng, F. Chu and Y. He, Vibration Signal Analysis and Feature Extraction Based on Reassigned Wavelet Scalogram, Journal of Sound and Vibration 253 (2002), 1087-1100.

[22] Z. Peng, Y. He, Z. Chen and F. Chu, Identification of the Shaft Orbit for Rotating Machines Using Wavelet Modulus Maxima, Mechanical Systems and Signal Processing 16 (2002), 623-635.

[23] P. Pennacchi and A. Vania, Use of the Orbit Shape Analysis to Detect Faults in Rotating Machines, Proc. of DINAME - X Symposium on Dynamic Problems of Mechanics, 2003, Ubatuba (Brasil), 275-281.

[24] R. Platz and R. Markert, Fault Models for On-line Identification of Malfunctions in Rotor Systems, Proc. of the 4 h Int. Conference on Acoustical and Vibratory Surveillance Methods and Diagnostic Techniques, 2001, Compègne (France), 435-446.

[25] S. Prabhakar, A.S. Sekhar and A.R. Mohanty, Vibration Analysis of a Misaligned Rotor-Coupling-Bearing system passing through the critical speed, Proc Instn Mech Engrs 215 (2001) part C, 1417-1428. 
[26] J.S. Rao and A. Chawla, Analytical and experimental investigation on misaligned rotors, ISROMAC-9 Conference, 2002, Honolulu (Hawaii), pp. 1-8.

[27] N.F. Rieger and S. Zhou, Development and verification of transfer matrix unbalance response procedure for three-level rotor-foundation systems, Journal of Vibration and Acoustics 120 (1998), 240-251.

[28] A.S. Sekhar and B.S. Prabhu, Effects of Coupling Misalignment on Vibrations of Rotating Machinery, Journal of Sound and Vibration 185 (1995), 655-671.

[29] M. Smart, M.I. Friswell, A.W. Lees and U. Prells, Estimating turbogenerator foundation parameters, Proc Instn Mech Engrs 212 (1998), part C, 635-665.

[30] A.T. Tapia and K.L. Cavalca, Modelling Effect of Flexible and Rigid Couplings in Mechanical Systems, Proc. of IFToMM-6th International Conference on Rotor Dynamics, 2002, Sydney (Australia), pp. 420-428

[31] A. Vania and P. Pennacchi, Experimental and theoretical application of fault identification measures of accuracy in rotating machine diagnostics, Mechanical Systems and Signal Processing 18 (2004), 329-352.

[32] V. Wowk, Machine Vibration: Alignment, Mc Graw-Hill, New York, 2000. 

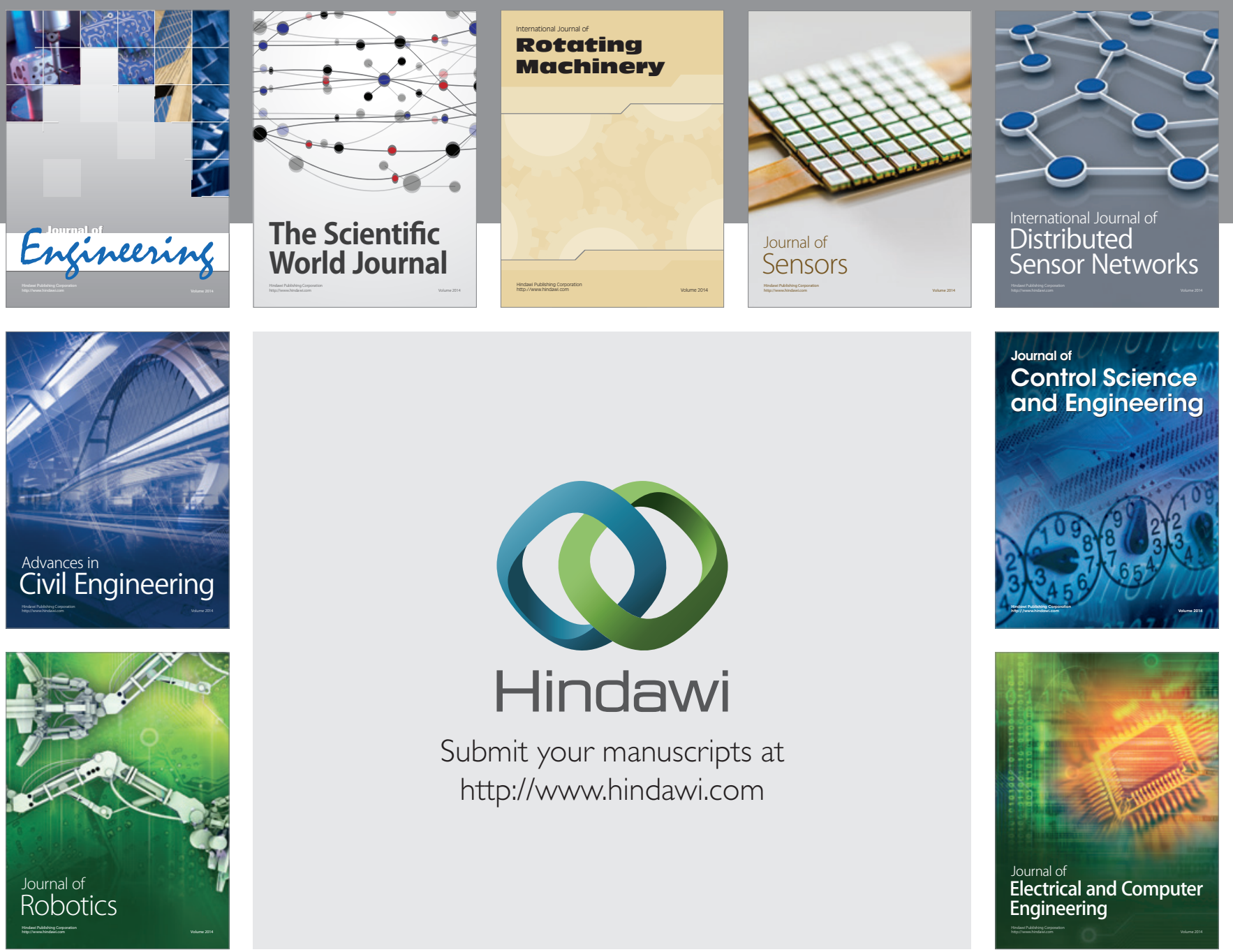

Submit your manuscripts at

http://www.hindawi.com
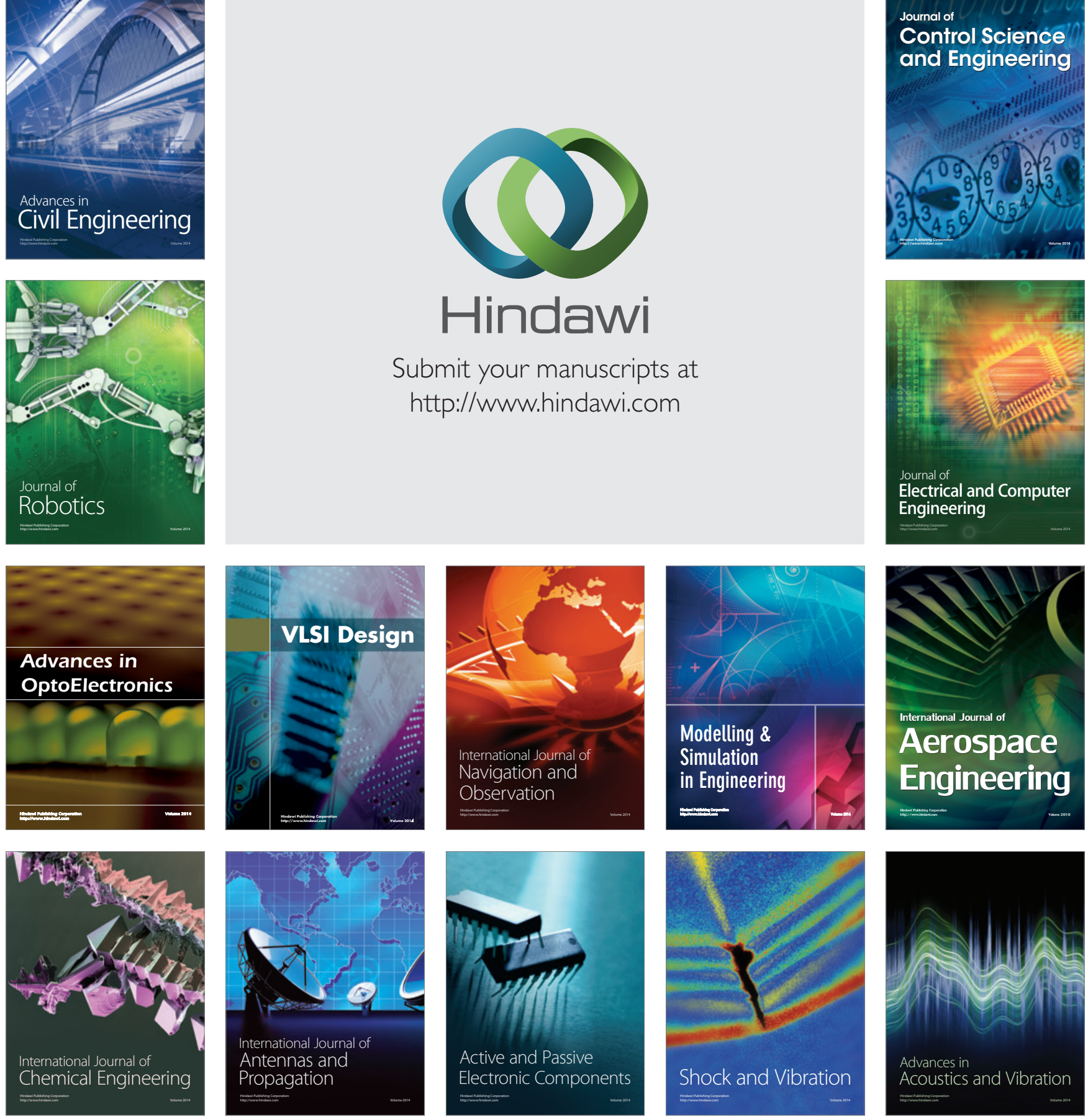